Quantum Field Theory Under the Influence

of External Conditions (QFEXT11)

International Journal of Modern Physics: Conference Series

Vol. 14 (2012) 485-495

(C) World Scientific Publishing Company

DOI: $10.1142 / \mathrm{S} 2010194512007611$

\title{
STOCHASTIC QUANTIZATION AND CASIMIR FORCES: PISTONS OF ARBITRARY CROSS SECTION
}

\author{
PABLO RODRIGUEZ-LOPEZ and RICARDO BRITO \\ Departamento de Física Aplicada I and GISC, Universidad Complutense, 28040 Madrid, Spain \\ brito@fis.ucm.es \\ RODRIGO SOTO \\ Departamento de Fúsica, FCFM, Universidad de Chile, Casilla 487-3, Santiago, Chile \\ rsoto@dfi.uchile.cl
}

Published 28 July 2012

\begin{abstract}
Recently, a method based on stochastic quantization has been proposed to compute the Casimir force and its fluctuations in arbitrary geometries. It relies on the spectral decomposition of the Laplace operator in the given geometry. Both quantum and thermal fluctuations were considered. Here we use this method to compute the Casimir force on the plates of a finite piston of arbitrary cross section. Asymptotic expressions valid at low and high temperatures, as well as short and long distances are obtained. The case of a piston with triangular cross section is analyzed in detail. The regularization of the divergent stress tensor is described.
\end{abstract}

Keywords: Stochastic quantization; quantum-thermal; pistons.

\section{Introduction}

H.G. Casimir ${ }^{1}$ predicted in 1948 the existence of an attractive force between two perfectly conducting parallel plates due to quantum fluctuations of the vacuum ${ }^{2}$. Advanced experimental setups allow one to measure such forces for geometries other than the two parallel plates ${ }^{3}$. However, techniques to calculate forces for complicated geometries, beyond the usual ones with high degrees of symmetry, were scarce until Ref. 4 introduced a method to calculate electromagnetic (EM) Casimir forces. The method is based on a multiscattering calculation and has been successfully applied to many configurations, such as plates, cylinders, spheres, wedges, etc. ${ }^{5}$

Casimir forces have their origin in fluctuations of EM fields. Already in 1956, Lifshitz ${ }^{6}$ used the concept of fluctuations (in this case of the electric currents inside a metal) to compute such forces. In Ref. 7 a new method to compute Casimir forces has been derived by us. It is based on the stochastic quantization approach developed by Parisi and $\mathrm{Wu}^{8}$. A Langevin equation for a given field subjected to thermal fluctuations is constructed in a non-physical, pseudo-time, such that it 
reproduces the probability distribution for the field in a thermal bath. Using the formalism developed in Ref. 9 to compute fluctuation-induced forces starting from a stochastic equation, the Casimir force of the electromagnetic field can be obtained. The resulting method has the advantage that one can compute the Casimir force directly and can be applied to compute torques and stresses over extended bodies as well. Also, the force fluctuations are obtained properly regularized and it was shown that the force variance on a piston geometry is finite and twice the square of the force ${ }^{7}$.

In this manuscript we apply this method to the computation of the force on pistons of arbitrary cross section. The text is organized as follows. Section 2 presents a summary of the procedure to obtain the Casimir force using the stochastic quantization method. An analysis of the divergence of the stress tensor is performed and a force regularization method is proposed. In Section 3 the method is applied to the case of the electromagnetic Casimir force on pistons. The decomposition in transverse electric and transverse magnetic modes is described resulting in an expression for the force on the plates in terms of the eigenvalues of the 2D Laplacian on the piston cross section. The force regularization is analyzed numerically and treated analytically, resulting in absolutely convergent expressions. Asymptotic formulas are provided. The case of pistons with polygonal cross section is analyzed in section 4 and the use of the method is illustrated by computing the force on pistons with a triangular cross section. Finally, conclusions are in Section 5.

\section{General formalism}

Here we summarize the formalism developed in Ref. 7. The Casimir force can be obtained averaging the stress tensor over the probability distribution of the quantum field. The stochastic quantization method of Parisi and $\mathrm{Wu}^{8,10}$ provides a stochastic equation in a pseudo time $s$ that reproduces the full probability distribution in space, $\mathbf{r}$, and Wick-rotated time, $\tau=i t$, of a quantum field at temperature $T$. In the case of a scalar bosonic field of zero mass $\phi$, such stochastic equation reads

$$
\partial_{s} \phi(\tau, \mathbf{r} ; s)=\left(\frac{1}{c^{2}} \frac{\partial^{2}}{\partial \tau^{2}}+\nabla^{2}\right) \phi+\eta(\tau, \mathbf{r} ; s)
$$

where $\eta(\tau, \mathbf{r} ; s)$ is the noise term, mimicking the source of fluctuations for the field $\phi$. It is white noise, $\delta$-correlated in $\tau, \mathbf{r}$, and $s$, with intensity $2 k_{B} T$. Equation (1) can be solved by diagonalization. The spatial part requires the diagonalization of the Laplacian in the geometry with the appropriate boundary conditions (BC),

$$
\nabla^{2} f_{n}(\mathbf{r})=-\lambda_{n}^{2} f_{n}(\mathbf{r})
$$

and the temporal part involves the Matsubara frequencies $\omega_{m}=2 \pi m / \beta \hbar c, m \in \mathbb{Z}$, which we have divided by $c$ to further simplify the notation and in order to put on equal foot the temporal and spatial contributions.

If the stress tensor is a bilinear function of the field (as is the case in electromagnetism), $\mathbb{T}=\mathbb{T}[\phi, \phi, \mathbf{r}]$, which defines the stress tensor operator $\mathbb{T}$, then the 
average over the fluctuations can be computed by decomposing the field $\phi$ into its eigenfunctions, to obtain (see details in Ref. 7):

$$
\langle\mathbb{T}(\mathbf{r})\rangle=\frac{1}{\beta} \sum_{n m} \frac{\mathbb{T}_{n n}(\mathbf{r})}{\lambda_{n}^{2}+\omega_{m}^{2}},
$$

where $\mathbb{T}_{n n}(\mathbf{r})=\mathbb{T}\left[f_{n}, f_{n}^{*}, \mathbf{r}\right]$. The sum over the Matsubara frequencies, $\omega_{m}$, gives,

$$
\langle\mathbb{T}(\mathbf{r})\rangle=\frac{\hbar c}{2} \sum_{n} \frac{\mathbb{T}_{n n}(\mathbf{r})}{\lambda_{n}}\left[1+\frac{2}{e^{\beta \hbar c \lambda_{n}}-1}\right] .
$$

Finally, to obtain the Casimir force over a certain body, the stress tensor must be integrated over the surface $\Omega$ that defines the object

$$
\mathbf{F}_{C}=\oint_{\Omega}\langle\mathbb{T}(\mathbf{r})\rangle \cdot d \mathbf{S} .
$$

This expression allows us to compute the quantum Casimir force, including the effects of a finite, nonvanishing temperature, in terms of the eigenvalues and eigenfunctions of the Laplace operator.

\subsection{Regularization}

Let us note that, as it occurs in most of the calculations of the Casimir Force, the expression for $\langle\mathbb{T}(\mathbf{r})\rangle$ in Eq. (4) is generally divergent at every point of space $\mathbf{r}$ if the sum over eigenvalues runs to infinity. Indeed, an estimation can be made by considering Fourier modes with wavevector $\mathbf{k}$. The eigenvalues of the Laplacian grow as $\lambda^{2} \sim k^{2}$ and the contribution of each mode to the stress tensor in the case of Dirichlet BC (as it is the case in electromagnetism) is also $\mathscr{T}_{k} \sim k^{2}$. Substitution into Eq. (4) results in a divergent expression for the stress tensor at every point, in particular at the object boundary.

However, the expression for the force, which is obtained by integrating over the surface of the body, Eq.(5), is finite. This means that the integration over the body regularizes the divergences of the averaged stress tensor. If we assume that such regularization is carried out mode by mode, we can interchange the integration over the surface and the summation over eigenvalues to obtain,

$$
\mathbf{F}_{C}=\frac{\hbar c}{2} \sum_{n} \frac{1}{\lambda_{n}}\left[1+\frac{2}{e^{\beta \hbar c \lambda_{n}}-1}\right] \oint_{\Omega} \mathbb{T}_{n n}(\mathbf{r}) \cdot d \mathbf{S}
$$

which is a finite result. Therefore, the interchange of the integral and summation regularizes the Casimir force, avoiding the use of ultraviolet cutoffs. Other regularizations, which in some cases may lead to non-universal forces or fluctuations, are, for instance, the subtraction of the vacuum stress tensor ${ }^{11}$ or by averaging the stress tensor over a finite area or a finite time ${ }^{12}$. Having regularized the divergences, Eq. (6) provides a new expression to calculate Casimir forces for a given geometry by diagonalizing the Laplace operator. Then, this approach is suitable for numerical calculations of Casimir forces in complicated, realistic geometries. Moreover, this 


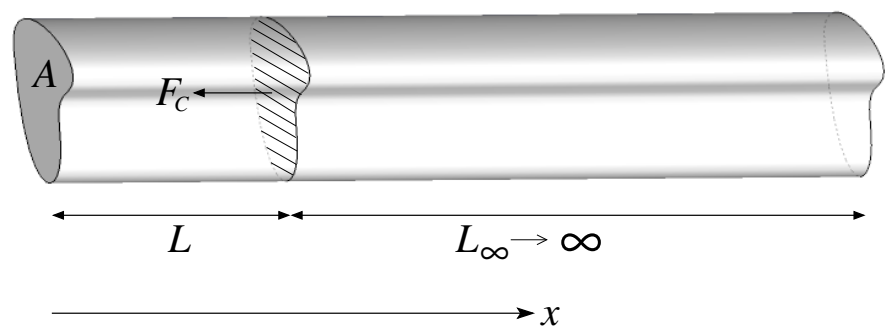

Fig. 1. Geometry of the considered problem. The Casimir force is evaluated for the plate of area $A$ and general geometry. The net force is obtained as the force exerted by a plate at distance $L$ and another plate at distance $L_{\infty} \rightarrow \infty$. The cylinder is oriented along the $x$ axis.

method leads to the force directly, not as a difference of the free energy with respect to a reference state, which in some configurations may be difficult to establish. Other authors have obtained formulas for the free energy in terms of the eigenvalues of the spatial operator, but not for the force ${ }^{13,14}$.

The expression for the Casimir force, Eq. (6), allows one to evaluate the quantum limit (by setting the temperature equal to zero) and the classical limit

$$
\lim _{T \rightarrow 0} \mathbf{F}_{C}=\frac{\hbar c}{2} \sum_{n} \frac{1}{\lambda_{n}} \oint_{\Omega} \mathbb{T}_{n n}(\mathbf{r}) \cdot d \mathbf{S} ; \quad \lim _{\hbar \rightarrow 0} \mathbf{F}_{C}=k_{B} T \sum_{n} \frac{1}{\lambda_{n}^{2}} \oint_{\Omega} \mathbb{T}_{n n}(\mathbf{r}) \cdot d \mathbf{S}
$$

The convergence of these expressions depend on the sum and integral exchange. The two limits, quantum and thermal, show that the driving force of the fluctuations has different origin. In the first case, the presence of the factor $\hbar$ indicates the quantum nature of the fluctuations, whereas in the second case, the factor $k_{B} T$ reveals its thermal origin.

\section{Electromagnetic Casimir force for a piston of arbitrary cross section}

\subsection{Electromagnetic Casimir force}

Let us consider the geometry depicted in Fig. 1. It consists of a piston of area $A$, but general shape, made of a perfectly conducting metal surface ${ }^{15}$. Two flat conducting plates of the same cross section of the piston are placed at a distance $L$ apart along the $x$ direction. The plates are perpendicular to the surface of the cylinder. We calculate the Casimir force, $F_{C}$, for this plate by evaluating the expression in Eq. (4) for $\langle\mathbb{T}(\mathbf{r})\rangle$ and later integrating over the surface. In order to obtain a finite result, we need a third auxiliary plate located at infinite distance, $L_{\infty} \rightarrow \infty$.

First, we solve the eigenvalue problem for the EM field and then apply Eqs. (3) and (5) to obtain the force. For the EM field the normal component of the stress tensor reads $\mathbb{T}_{x x}=E_{x}^{2}+B_{x}^{2}-\frac{1}{2} \mathbf{E}^{2}-\frac{1}{2} \mathbf{B}^{2}$, with $\mathrm{BC}, \mathbf{E} \times \mathbf{n}=\mathbf{0}$ and $\mathbf{B} \cdot \mathbf{n}=0$, where $\mathbf{n}$ is the surface normal vector. In this geometry, the EM field can be decomposed 
into transverse electric (TE) and transverse magnetic (TM) modes, which are discussed independently ${ }^{16}$. For the TM modes, the magnetic field is transverse to the $x$ direction, and the vector potential $\mathbf{A}$ can be written as

$$
\mathbf{A}=\left(-C \nabla_{\perp}^{2} D, \partial_{y} D \partial_{x} C, \partial_{z} D \partial_{x} C\right) e^{-i \omega t} .
$$

Here the fields $C(x)$ and $D\left(\mathbf{r}_{\perp}\right)$ satisfy

$$
\begin{aligned}
\partial_{x}^{2} C(x) & =-k_{x}^{2} C(x), \quad(\text { Neumann } \mathrm{BC} \text { on } x=0, L) \\
\nabla_{\perp}^{2} D_{n}\left(\mathbf{r}_{\perp}\right) & =-\lambda_{n}^{2} D_{n}\left(\mathbf{r}_{\perp}\right), \quad(\text { Dirichlet BC on } \mathcal{S}),
\end{aligned}
$$

where $\mathcal{S}$ is the surface of the cylinder and $\mathbf{r}_{\perp}=(y, z)$. For the TE set, the electric field is transverse to $x$, so the vector potential is $\mathbf{A}=\left(0,-S \partial_{z} N, S \partial_{y} N\right) e^{-i \omega t}$, where the functions $S(x)$ and $N\left(\mathbf{r}_{\perp}\right)$ satisfy Eqs. (9) with the opposite BC: Dirichlet for $S(x)$ and Neumann for $N\left(\mathbf{r}_{\perp}\right)$. However, in this case, the constant eigenfunction (with eigenvalue $\lambda_{n}^{2}=0$ ) must be excluded as it gives $\mathbf{A}=\mathbf{0}$ and then $\mathbf{E}=\mathbf{B}=\mathbf{0}$.

Next we substitute the TE modes into the expression for the stress tensor and integrate over one side of the plates. It gives, after a long but straightforward calculation, the Casimir force as

$$
\int_{1 \text { side }}\left\langle\mathbb{T}_{x x}^{T E}\right\rangle d S_{x}=\frac{1}{\beta L} \sum_{m \in \mathbb{Z}} \sum_{n_{x}=1}^{\infty} \sum_{n} \frac{k_{x}^{2}}{\omega_{m}^{2}+k_{x}^{2}+\lambda_{n}^{2}},
$$

where $k_{x}^{2}=\left(n_{x} \pi / L\right)^{2}$. For the TM modes, one obtains exactly the same expression, but the $\lambda_{n}^{2}$ are the eigenvalues of the two-dimensional (2D) Laplacian with Neumann BC. We will denote the complete set of eigenvalues of the Laplacian with Neumann (excluding the zero eigenvalue) and Dirichlet BC by the index $p$. The expression above is the equivalent of Eq. (3) when the spectrum can be split into a longitudinal and transversal part, that is $\lambda_{n}^{2}=k_{x}^{2}+\lambda_{p}^{2}$. The sum over the Matsubara frequencies can be performed as in (4). In the limit of vanishing temperature $\left(\omega_{1}=2 \pi k_{B} T / \hbar c \rightarrow\right.$ 0) this reduces to

$$
\lim _{T \rightarrow 0} \int_{1 \text { side }}\left\langle\mathbb{T}_{x x}^{\text {total }}\right\rangle d S_{x}=\hbar c \pi^{2} \sum_{n_{x}=1}^{\infty} \sum_{p} \frac{n_{x}^{2}}{L^{3} \sqrt{n_{x}^{2} \pi^{2} / L^{2}+\lambda_{p}^{2}}} .
$$

\subsection{Regularization}

The series (11) and the one at finite temperature are divergent, but the net Casimir force, which is the difference between the force exerted by the plate at distance $L$ and the plate at $L_{\infty} \rightarrow \infty$ (see Fig. 1), is finite. To apply the regularization method described in Section 2.1, an eigenvalue ordering method is used to obtain the contribution to the total force (on both sides) of each mode. That is, a kernel 
ordering method is considered. In the case of vanishing temperature this reads

$$
F_{T=0}=\lim _{Q \rightarrow \infty} \hbar c \pi^{2} \sum_{n_{x}=1}^{\infty} \sum_{p} n_{x}^{2}\left[\frac{K\left[\left(\frac{n_{x}^{2} \pi^{2}}{L^{2}}+\lambda_{p}^{2}\right) / Q\right]}{L^{3} \sqrt{\frac{n_{x}^{2} \pi^{2}}{L^{2}}+\lambda_{p}^{2}}}-\frac{K\left[\left(\frac{n_{x}^{2} \pi^{2}}{L_{\infty}^{2}}+\lambda_{p}^{2}\right) / Q\right]}{L_{\infty}^{3} \sqrt{\frac{n_{x}^{2} \pi^{2}}{L_{\infty}^{2}}+\lambda_{p}^{2}}}\right]
$$

where $K(x)$ is the regularizing kernel that must satisfy $\lim _{x \rightarrow 0} K(x)=1$ and $\lim _{x \rightarrow \infty} K(x)=0$, and $Q$ is the cutoff eigenvalue.

To study the convergence of the kernel regularization (12) we consider the case of a circular plate. In this case, the eigenvalues are the zeros of the Bessel function $J_{\nu}(r)$ and its derivative. The sum can be done numerically, and Fig. 2 shows $F_{C}$ as a function of $Q$. It is observed that although the force on both sides diverge when $Q \rightarrow \infty$ (see inset of Fig. 2), the net force on the plate converges to a finite value.

For a general piston geometry, the force can be regularized analytically using the Chowla-Selberg summation formula to rewrite the sum over the variable $n_{x}$ in Eq. $(10)^{17}$. This formula extracts the divergent, $L$-independent part of the summation, which cancels when the integral in Eq. (10) is performed for both sides of the piston. Equivalently, the $L$-independent contribution is the same for the second

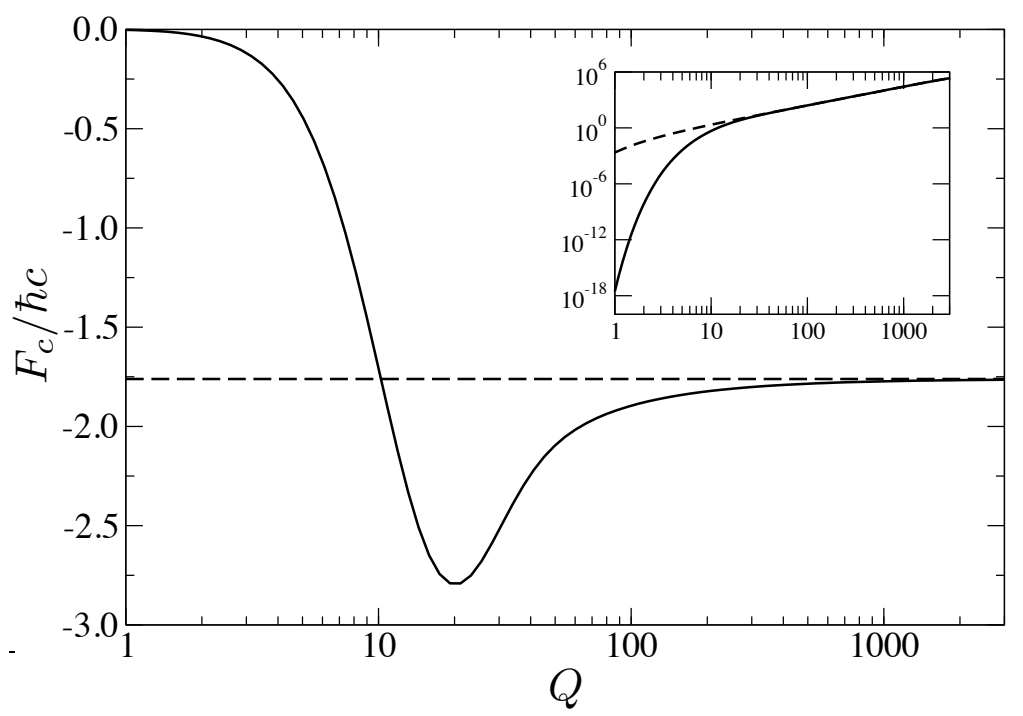

Fig. 2. Convergence of the Casimir force on a circular plate using the kernel regularization method with $K(x)=e^{-x}$. The circle radius is $R=1$, the interior plate distance is $L=0.5$ and the exterior plate distance is $L_{\infty}=100.0$. The number of eigenvalues used in the evaluation is 3000 , being $\lambda_{3000}^{2} \approx 77.5$. Main figure: net force $(12)$ as a function of the cutoff value $Q$ (solid line) and the asymptotic value obtained using the analytic regularization (14) (dashed line). Inset: force on the interior (solid line) and exterior (dashed line) sides of the plate as a function of $Q$. 
plate at distance $L$ or at distance $L_{\infty}$, resulting in

$$
F_{C}=-\frac{1}{\beta} \sum_{p} \sum_{m \in \mathbb{Z}} \frac{\sqrt{m^{2} \Lambda^{2}+\lambda_{p}^{2}}}{e^{2 L \sqrt{m^{2} \Lambda^{2}+\lambda_{p}^{2}}}-1} .
$$

Here, $\Lambda=2 \pi / \beta \hbar c$ is the inverse thermal wavelength. This expression gives the finite or regularized Casimir force between two plates at distance $L$, valid for any cross section and temperature. The precise geometry of the plates enters into the double set of eigenvalues of the Laplacian $\lambda_{p}^{2}$.

In the limit of vanishing temperature the sum over $m$ in Eq. (13) can be rigorously replaced by an integral, resulting in

$$
F_{T=0}=-\frac{\hbar c}{2 \pi} \sum_{p} \sum_{n=1}^{\infty} \lambda_{p}^{2}\left[K_{0}\left(2 n L \lambda_{p}\right)+K_{2}\left(2 n L \lambda_{p}\right)\right] .
$$

Here $K_{\alpha}(x)$ is the modified Bessel function of order $\alpha$. Reference 13 obtained a formula for the free energy of the configuration considered here, that, after differentiation with respect to the distance between the plates, leads to the force above.

In a similar fashion, we can calculate the thermal Casimir force when $\hbar \rightarrow 0$. Then, in Eq. (13), only the term with $m=0$ is different from zero, resulting in

$$
F_{\hbar=0}=-\frac{1}{\beta} \sum_{p} \frac{\lambda_{p}}{e^{2 L \lambda_{p}}-1} .
$$

\subsection{Short and long distances}

For short distances, the summation over $p$ can be calculated without explicitly knowing the shape of the piston and the eigenvalues. For simple cross sections, the eigenvalues, $\lambda_{p}^{2}$, scale with the inverse of the square of the typical size of the piston, because of dimensional arguments. So, for distances $L$ much smaller than the section of the piston, we can replace the sum over the eigenvalues, $\lambda_{p}^{2}$, by an integral. In doing that step, we need the asymptotic expression for the density of states of the Laplacian in two dimensions, that reads (for both Dirichlet or Neumann $\mathrm{BC}^{18}$ ):

$$
\rho_{\eta}(\lambda)=[2 \lambda A+\eta P] \theta(\lambda) / 4 \pi+\chi \delta(\lambda),
$$

where $A$ is the piston area, $P$ its diameter, $\eta=1(-1)$ for Dirichlet (Neumann) and $\chi$ is a measure of the perimeter curvature defined as follows. For a piecewise continuous boundary,

$$
\chi=\frac{1}{24} \sum_{i}\left[\frac{\pi}{\alpha_{i}}-\frac{\alpha_{i}}{\pi}\right]+\frac{1}{12 \pi} \sum_{j} \int_{\gamma_{j}} \kappa\left(\gamma_{j}\right) d \gamma_{j},
$$

where $d \gamma_{j}$ are the line elements of the continuous segments, $\kappa\left(\gamma_{j}\right)$ the finite curvature at each point of the boundary, and $\alpha_{i}$ is the angle of each vertex joining contiguous segments. 
When integrating using the density of states, the zero eigenvalue of the Neumann BC must be excluded. Therefore, the expression (13) in the limit of short distances can be computed as

$$
F_{C}=-\frac{1}{\beta} \sum_{\eta=-1,1} \int_{0}^{\infty} d \lambda \rho_{\eta}(\lambda) \sum_{m \in \mathbb{Z}} \frac{\sqrt{m^{2} \Lambda^{2}+\lambda^{2}}}{e^{2 L \sqrt{m^{2} \Lambda^{2}+\lambda^{2}}}-1}+\lim _{\lambda \rightarrow 0} \frac{1}{\beta} \sum_{m \in \mathbb{Z}} \frac{\sqrt{m^{2} \Lambda^{2}+\lambda^{2}}}{e^{2 L \sqrt{m^{2} \Lambda^{2}+\lambda^{2}}}-1},
$$

where the total density of states (Dirichlet plus Neumann) has been considered. After a lengthy but straightforward calculation

$$
\begin{aligned}
F_{C}= & -k_{B} T \frac{A}{4 \pi L^{3}} \sum_{m=0}^{\infty}{ }^{\prime}\left[\operatorname{Li}_{3}\left(e^{-2 L m \Lambda}\right)+2 L m \Lambda \operatorname{Li}_{2}\left(e^{-2 L m \Lambda}\right)+2 L^{2} m^{2} \Lambda^{2} \operatorname{Li}_{1}\left(e^{-2 L m \Lambda}\right)\right] \\
& -2 k_{B} T(2 \chi-1) \sum_{m=1}^{\infty} \frac{m \Lambda}{e^{2 L m \Lambda}-1}
\end{aligned}
$$

where the apostrophe in the sum indicates that the term $m=0$ should be multiplied by a factor $1 / 2$ and $\operatorname{Li}_{s}(z)$, are polylog functions. Note that the force depends on the area and the curvature, but not on the perimeter of the piston.

Taking the limit of low temperature the quantum Casimir force is

$$
F_{T=0}^{\text {near }}=-\frac{\hbar c A \pi^{2}}{240 L^{4}}-\frac{\hbar c(2 \chi-1) \pi}{24 L^{2}} .
$$

The first term is the well-known result of the EM Casimir force for infinite parallel plates $^{1}$, and the second term is a contribution of the curvature.

In the thermal case, of high temperatures, the force only depends on the area,

$$
F_{\hbar=0}^{\text {near }}=-\frac{k_{B} T A}{4 L^{3}} \zeta(3)
$$

In the opposite long distance limit, when $L$ is much larger than the typical size of the plate only the smallest eigenvalue $\lambda_{1}^{2}$ contributes to the sum, with the result

$$
F_{T=0}^{\mathrm{far}}=-\frac{\hbar c}{2 \sqrt{\pi L}} g_{1} \lambda_{1}^{3 / 2} e^{-2 L \lambda_{1}}, \quad F_{\hbar=0}^{\mathrm{far}}=-k_{B} T g_{1} \lambda_{1} e^{-2 L \lambda_{1}},
$$

where $g_{1}$ the degeneracy of $\lambda_{1}$.

\section{Polygonal pistons}

A particularly interesting case is that of pistons with a regular polygonal cross section. If $N$ is the number of sides of the polygon, and $R_{c}$ is the exterior (circumscrit) radius of it, the polygon area and curvature parameter are

$$
A=\frac{\sin (2 \pi / N)}{2 \pi / N} \pi R_{c}^{2}, \quad \chi=\frac{N-1}{6(N-2)} .
$$

Using the value of the first eigenvalue, the far and near regimes of the force can be easily computed. 
To illustrate the intermediate behavior and the transition from the near to the far regime, we study the case of a equilateral triangle of side $a$. The relevant quantities for the computation in the asymptotic regimes are ${ }^{19}$

$$
A=a^{2} \sqrt{3} / 4 ; \quad \chi=1 / 3 ; \quad \lambda_{1}^{2}=16 \pi^{2} / 9 a^{2} \text { (Neumann) } ; \quad g_{1}=2 .
$$

At intermediate distances, that is, $L$ comparable to the size of the plates, one must solve the eigenvalue problem, either numerically using finite element methods for a general geometry, or analytically for special geometries as in the present case ${ }^{19}$. The dependence with the side of the triangle is $\lambda_{p}^{2}(a)=\lambda_{p}^{2}(a=1) / a^{2}$, so in the quantum limit the Casimir force as expressed in Eq. (14) is a function of $L / a$ when the force is multiplied by $a^{2}$, while in the thermal limit the Casimir force Eq. (15) is a function of $L / a$ when the force is multiplied by $a$.

Figure 3 (left panel), shows the Casimir force in the quantum limit, Eq. (14), for an equilateral triangle of side $a$. To compare, five results are presented: $(i)$ the full computation using an extensive list of eigenvalues $(N=4000$, that ensures convergence for $L / a \geq 0.03$ ) that we consider as the true value; (ii,iii) the asymptotic expression for near plates (19) with and without the curvature correction; $(i v)$ the asymptotic expression for far plates $(21)$; and $(v)$ a partial sum considering only the first 20 eigenvalues. A similar comparison in the thermal limit is presented in the same figure (right panel). The analysis of both cases, quantum and thermal, is qualitatively similar. When the plates are separated, only a small number of eigenvalues is needed to obtain the force (20 eigenvalues for $L / a>0.2)$. At smaller distances, more eigenvalues would be needed, but there is an excellent match with the asymptotic expansion at short distances. The curvature correction in the quantum limit allows us to extend its validity up to $L / a \approx 0.4$.

\section{Conclusions}

The new method described in Ref. 7 to compute Casimir forces using the stochastic quantization formalism has been used to calculate the force on pistons of arbitrary cross section. The method is quite simple, and it provides the force directly, instead of the free energy. It only requires the spectral decomposition of the Laplace operator in the given geometry, summation of the eigenvalues, and the integration of the eigenfunctions along the boundary of the object. Such integration of the eigenfunctions over the surface of the body leads to a regularization of the Casimir force, producing finite results for the force. Quantum and classical limits are recovered as well as intermediate results for finite temperatures. The electromagnetic Casimir force on pistons with arbitrary cross section is analyzed in detail. The computation of the 2D eigenvalue problem allows one to obtain the Casimir force in the whole range of separation distances. To demonstrate the method, the force on a piston of triangular cross section is computed numerically.

We thank J. Horowitz, A. Muñoz-Sudupe y A. Núñez for useful comments. P.R.L. and R.B. are supported by the Spanish projects MOSAICO, FIS2011-22644 and 

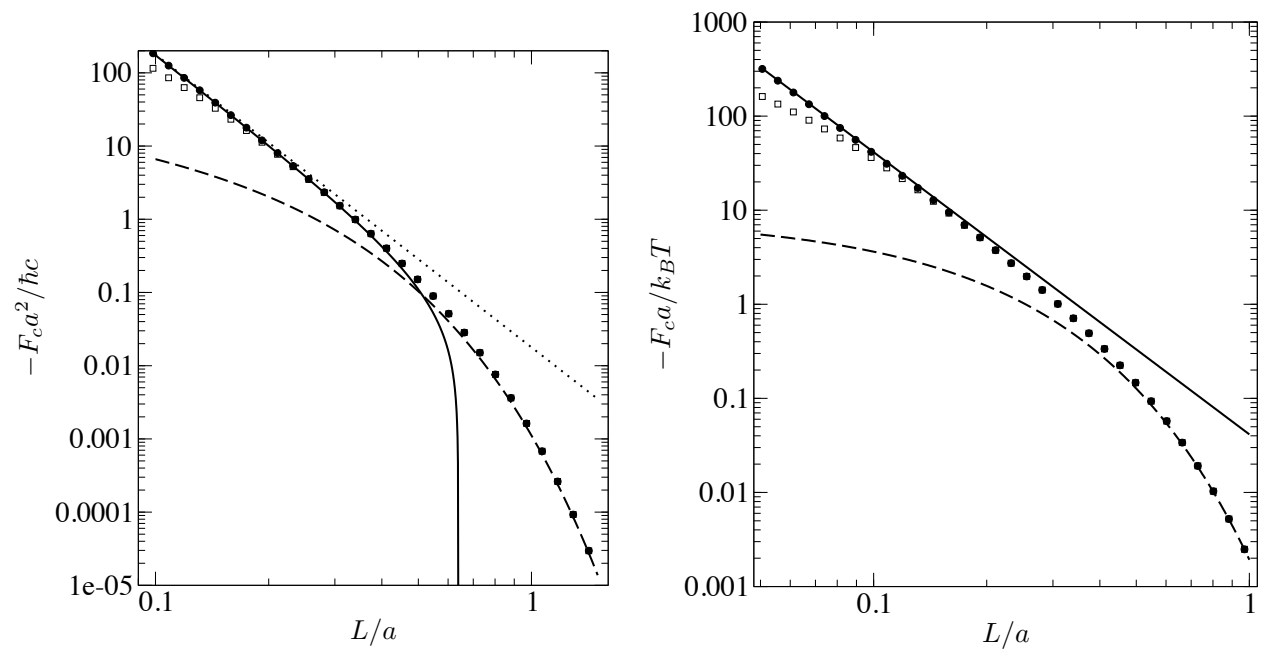

Fig. 3. Left: Quantum limit of the Casimir force for a piston of triangular cross section of side $a$ as a function of the distance $L$ between the plates. Solid circles are obtained summing over 4000 eigenvalues of the Laplacian, using Eq. (14). Open squares (overlapping with circles for $L / a>0.2$ ) are obtained summing only over 20 eigenvalues. Asymptotic expressions for near plates (19) without the curvature correction (dotted line), with the curvature correction (solid line), and the asymptotic expression for far plates (21) (dashed line). Right: Thermal limit of the Casimir force for the same configuration. Solid circles are obtained summing over 4000 eigenvalues of the Laplacian, using Eq. (15). Open squares are obtained summing only over 40 eigenvalues. Asymptotic expressions for near plates (20) (solid line) and the asymptotic expression for far plates (21) (dashed line).

MODELICO. P.R.-L.'s research is also supported by an FPU MEC grant. R.S. is supported by Fondecyt grant 1100100 and Proyecto Anillo ACT 127.

\section{References}

1. H.B.G. Casimir, Proc. K. Ned. Akad. Wet. 51, 793 (1948).

2. M. Bordag, G.L. Klimchitskaya, U. Mohideen, and V.M. Mostepanenko, Advances in the Casimir Effect, Oxford University Press, Oxford 2009.

3. G.L. Klimchitskaya, U. Mohideen, V.M. Mostepanenko, Rev. Mod. Phys. 81, 18271885 (2009)

4. T. Emig, et al., Phys. Rev. Lett. 99, 170403 (2007). S.J. Rahi, et al. Phys. Rev. D 80, 085021 (2009).

5. M.F. Maghrebi, et al., Proc. Natl. Acad. Sci. U.S.A. 108, 6867 (2011) and references therein.

6. E.M. Lifshitz, Sov. Phys. JEPT 2, 73 (1956).

7. P. Rodriguez-Lopez, R. Brito, and R. Soto, Europhys. Lett. 96, 50008 (2011).

8. G. Parisi and Y.-S. Wu, Sci. Sin. 24, 483 (1981). P.H. Damgaard and H. Hüffel, Phys. Rep. 152, 227 (1987).

9. P. Rodriguez-Lopez, R. Brito, and R. Soto, Phys. Rev. E 83, 031102 (2011).

10. M. Masujima, Path Integral Quantization and Stochastic Quantization, Springer Berlin 2009. 
11. C.-H. Wu, L.H. Ford, Phys. Rev. D 64, 045010 (2001). C.-H.Wu, C.-I Kuo, L.H. Ford, Phys. Rev. A 65, 062102 (2002).

12. G. Barton, J. Phys. A: Math. Gen. 24, 991 (1991). G. Barton, J. Phys. A: Math. Gen. 24, 5533 (1991).

13. V.N. Marachevsky, J. Phys. A: Math. Gen. 41, 164007 (2008).

14. E.K. Abalo, K.A. Milton, and L. Kaplan, Phys. Rev. D 82, 125007 (2010).

15. M.P. Hertzberg, et al. Phys. Rev. D 76, 045016 (2007).

16. J.D. Jackson, Classical Electrodynamics, 3rd ed. Wiley, 1999, chapter 8.3.

17. E. Elizalde, J. Phys. A: Math. Gen. 27, 3775 (1994). See also Eq. (A.3) of Ref. ${ }^{9}$.

18. H. Baltes, Spectra of Finite Systems, Birkhaüser Boston, 1980, Chapter VI.

19. B.J. McCartin, SIAM Review 45, 267 (2003). B. J. McCartin, Math. Probl. Eng. 8, 517 (2002). 